Research Article

\title{
Comparison of Respiratory Pathogen Detection in Upper versus Lower Respiratory Tract Samples Using the BioFire FilmArray Respiratory Panel in the Immunocompromised Host
}

\author{
Natalya Azadeh $\mathbb{D}^{1},{ }^{1}$ Kenneth K. Sakata ${ }^{D},{ }^{1}$ Ali Saeed, ${ }^{1}$ John J. Mullon, ${ }^{1}$ Thomas E. Grys, ${ }^{3}$ \\ Andrew H. Limper, ${ }^{1}$ and Matthew J. Binnicker ${ }^{2}$ \\ ${ }^{1}$ Department of Pulmonary and Critical Care Medicine, Mayo Clinic, Rochester, MN, USA \\ ${ }^{2}$ Department of Laboratory Medicine and Pathology, Mayo Clinic, Rochester, MN, USA \\ ${ }^{3}$ Division of Clinical Microbiology, Mayo Clinic, Scottsdale, AZ, USA
}

Correspondence should be addressed to Natalya Azadeh; azadeh.natalya@mayo.edu

Received 9 October 2017; Accepted 8 March 2018; Published 5 April 2018

Academic Editor: Dario Olivieri

Copyright (c) 2018 Natalya Azadeh et al. This is an open access article distributed under the Creative Commons Attribution License, which permits unrestricted use, distribution, and reproduction in any medium, provided the original work is properly cited.

Background. The FilmArray Respiratory Panel (FARP) (BioFire Diagnostics, Inc.) is a multiplex, polymerase chain reaction (PCR) technique that can detect 17 respiratory viruses and 3 bacterial targets in a single reaction. Immunocompromised hosts (ICH) with respiratory illnesses often undergo bronchoscopy with bronchoalveolar lavage (BAL). This prospective study aimed to evaluate the yield and concordance of NP and BAL FARP testing when performed on the same patient concurrently. Methods. From February to December 2016, 125 patients (100 ICH and 25 non-ICH) were enrolled. NP swabs and BAL samples were sent for FARP testing. Results. The yield of the BAL FARP among ICH and non-ICH was $24 \%(24 / 100)$ and $8 \%(2 / 25)$, respectively. The yield of positive NP swabs in ICH was $27 \%(27 / 100)$ versus $4 \%(1 / 25)$ in non-ICH. The majority of patients $(89 \% ; 111 / 125)$ had concordant results between NP and BAL specimens. Of the $24 \mathrm{ICH}$ patients who had a positive BAL FARP, the majority (79\%) had the same pathogen detected from the NP swab. Conclusion. The FARP may be useful in the ICH. Given the high concordance, in patients whom a pathogen is identified on the NP FARP, a FARP performed on BAL will likely yield the same result. However, if the NP FARP is negative, performing the test on a BAL sample may have an incremental yield.

\section{Introduction}

The FilmArray ${ }^{\circledR}$ Respiratory Panel (FARP) (BioFire Diagnostics, Salt Lake City, UT) is a multiplex PCR assay that can rapidly ( $\sim 60 \mathrm{~min})$ detect 17 common respiratory viruses and 3 bacterial targets in a single reaction [1]. Published studies have shown that multiplex PCR panels are more rapid and sensitive compared to routine culture and antigen detection methods [1-8]. Although multiplex panels are rapid and accurate, the high cost to the laboratory and patient may limit their use in resource-limited settings.

For the majority of immunocompetent patients with respiratory symptoms, diagnostic testing may not be required. If testing is completed, it is usually done on an easily accessible specimen type (e.g., throat swab or nasopharyngeal [9]
(NP) swab) and is generally limited to targeted pathogens (e.g., influenza virus). However, in patients who are immunocompromised or critically ill, a rapid, syndromic panel may be of benefit. Furthermore, patients with persistent or progressive symptoms may require a more extensive diagnostic evaluation, including bronchoscopy with collection of bronchoalveolar lavage (BAL) fluid. The utility of FARP testing using NP swabs and BAL specimens collected from immunocompromised hosts ( $\mathrm{ICH}$ ) has been investigated $[2,7,10]$. Our group completed a previous, retrospective study analyzing the FARP in patients who underwent bronchoscopy with BAL for evaluation of a respiratory illness. The results of the multiplex panel on BAL were compared to those of testing on a NP swab that had been collected within 7 days of the bronchoscopy. This study demonstrated high 
TABLE 1: Reason for immunocompromised status.

\begin{tabular}{lr}
\hline Condition leading to immunosuppression & Number (\%) of patients \\
\hline HSCT & $49(49.0)$ \\
SOT & $27(27.0)$ \\
Connective tissue disease on immunosuppression & $10(10.0)$ \\
Chronic prednisone therapy & $8(8.0)$ \\
Cytotoxic chemotherapy & $3(3.0)$ \\
HIV/AIDS & $2(2.0)$ \\
CVID & $1(1.0)$ \\
Total & $100(100.0)$ \\
\hline
\end{tabular}

HSCT, hematopoietic stem cell transplant recipient; SOT, solid organ transplant recipient; CVID, common variable immune deficiency.

concordance $(77 \%)$ between the FARP completed on NP swabs and BAL specimens [10]. However, the prior study was limited by the fact that the NP swab and BAL fluid were collected at different time points, and the data were analyzed mainly to compare overall concordance of the FARP between the two specimen types. In order to extend on these prior observations, we sought to prospectively evaluate the yield and concordance of the FARP using NP swabs and BAL fluid collected concurrently from ICH and non-ICH comparison group. This study was approved by the Mayo Clinic Institutional Review Board.

\section{Methods}

2.1. Patient Population. Between February and December 2016, 125 patients (100 ICH and 25 non-ICH) who underwent a bronchoscopy with BAL as part of their routine clinical care were enrolled in this study. Ages ranged between 26 and 85 years (mean 70 years), and 58\% were male. An immunocompromised status was defined as patients who were (1) recipients of systemic steroids (for $\geq 21$ days), disease-modifying agents (e.g., inhibitors of tumor necrosis factor), chemotherapy, or calcineurin inhibitors, (2) HIV positive, (3) solid organ or stem cell transplant recipients, and/or (4) positive for a lymphoproliferative or myelodysplastic syndrome (Table 1). ICH patients (e.g., lung transplant recipients) who were undergoing routine graft rejection surveillance transbronchial biopsies and BAL were also included in our analysis. They would also serve as an ICH control group to determine whether the FARP tested positive in asymptomatic ICH patients. Twenty-five patients who did not meet the criteria listed above were also included as part of a non-ICH comparison group. These were patients undergoing bronchoscopy with BAL to rule out infection but were not considered immunocompromised. Once enrolled, the patients' electronic medical records were reviewed and the following information was obtained: demographics (age and sex), presence and reason of immunosuppression, microbiology testing data from the BAL specimen (e.g., cultures and PCR), specifics of the respiratory illness (e.g., cough and wheezing), and other clinical or radiographic features. When the results of other microbiology studies (e.g., routine culture) were reviewed, only those results that led to an impact on patient management and were considered to be true pathogens were analyzed.
2.2. Specimen Collection. The protocol for collection and testing of specimens was approved by the institutional review board at our center. Patients provided informed consent and subsequently were sedated prior to bronchoscopy. Following sedation, a wire-shafted, BBL CultureSwab (Becton Dickinson, Franklin Lakes, NJ) was used to sample the posterior nasopharynx per routine protocol and subsequently placed in $3 \mathrm{~mL}$ of M5 viral transport media (Remel, Lenexa, KS). Next, a bronchoscopy with BAL was performed, in which $20 \mathrm{~mL}$ aliquots of normal saline were instilled into a subsegmental bronchus corresponding to an area of radiographic abnormality on CT. Forty $\mathrm{mL}$ of BAL fluid was recovered by immediate return suction. A 1-2 mL aliquot of the BAL specimen and the NP swab were transported to the laboratory, stored at $4^{\circ} \mathrm{C}$, and tested within 24 hours.

2.3. Routine Microbiology Testing. In addition to FARP testing, which was performed solely for study purposes, the following microbiology studies were completed on the BAL sample as part of each patient's routine clinical care: Gram stain, fungal smear, acid-fast smear, routine cultures (bacterial, fungal, viral, and mycobacterial), real-time PCR for influenza, respiratory syncytial virus (RSV), Pneumocystis jirovecii (PJP), adenovirus, and Legionella spp. The NP swab was collected solely for study purposes, and no other microbiology studies were conducted on this source.

2.4. FilmArray Respiratory Panel Testing. NP swabs in viral transport media were tested by the FilmArray Respiratory Panel according to the manufacturer's instructions. In addition, $0.3 \mathrm{~mL}$ of raw BAL fluid was loaded into a FilmArray v1.6 pouch and tested on the FilmArray 2.0 instrument (BioFire) following the same protocol used for NP swabs.

2.5. Statistical Analysis. All statistical analysis was done using comparison of proportions online chi-squared calculator (https://www.medcalc.org/calc/comparison_of_ proportions.php).

\section{Results}

Among 125 patients enrolled in this study, the multiplex panel was positive for at least one target in 28 (22\%) NP specimens and 26 (21\%) BAL samples. The majority (89\%; 
TABle 2: Concordance of the FilmArray Respiratory Panel when performed on paired NP swabs and BAL fluid specimens $(n=125)$.

Number (\%) of specimens yielding concordant FilmArray results

\begin{tabular}{lc}
\hline NP and BAL both positive & $20(16.0 \%)$ \\
NP and BAL both negative & $91(72.8 \%)$ \\
Total & $111(88.8 \%)$ \\
Number (\%) of specimens yielding discordant FilmArray results & \\
NP positive, BAL negative & $8(6.4 \%)$ \\
NP negative, BAL positive & $6(4.8 \%)$ \\
Total & $14(11.2 \%)$ \\
\hline
\end{tabular}

BAL, bronchoalveolar lavage; NP, nasopharyngeal.

TABle 3: Comparison of FilmArray Respiratory Panel using paired nasopharyngeal swab and BAL samples collected from immunocompromised hosts $(n=100)$.

\begin{tabular}{lrr}
$\begin{array}{l}\text { Number (\%) of NP swab samples with a FilmArray } \\
\text { result }\end{array}$ & \multicolumn{2}{c}{$\begin{array}{c}\text { Number (\%) of BAL specimens with a FilmArray } \\
\text { result }\end{array}$} \\
\hline $\begin{array}{l}\text { Positive } \\
\text { Negative }\end{array}$ & Positive & $8(8.0)^{\mathrm{b}}$ \\
\hline
\end{tabular}

BAL, bronchoalveolar lavage; NP, nasopharyngeal; ${ }^{a}$ rhinovirus/enterovirus $(n=9)$, parainfluenza type $3(n=2)$, coronavirus HKU1 ( $\left.n=1\right)$, coronavirus NL63 $(n=1)$, parainfluenza type $1(n=1)$, human metapneumovirus $(n=1)$, Bordetella pertussis $(n=1)$, influenza A H3 $(n=1)$, coronavirus OC43 and rhinovirus/enterovirus $(n=1)$, and adenovirus, coronavirus 229E, and rhinovirus/enterovirus $(n=1)$; ${ }^{b}$ rhinovirus/enterovirus $(n=6)$ and coronavirus HKU1 $(n=2)$; ${ }^{c}$ rhinovirus/enterovirus $(n=2)$, coronavirus HKU1 $(n=1)$, parainfluenza type $2(n=1)$, and respiratory syncytial virus $(n=1)$.

TABLE 4: Comparison of FilmArray respiratory panel using paired nasopharyngeal swab and BAL samples collected from immunocompetent hosts $(n=25)$.

\begin{tabular}{lcr}
\hline $\begin{array}{l}\text { Number (\%) of NP swab samples with a FilmArray } \\
\text { result }\end{array}$ & Number (\%) of BAL specimens with a FilmArray \\
result & Positive & Negative \\
\hline Positive & $1(4.0)^{\mathrm{a}}$ & $0(0.0)$ \\
Negative & $1(4.0)^{\mathrm{b}}$ & $23(92.0)$ \\
\hline
\end{tabular}

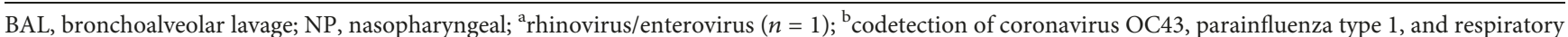
syncytial virus $(n=1)$.

$111 / 125)$ of paired NP and BAL samples had concordant results. Of these, $73 \%(91 / 125)$ were negative for all targets in both NP and BAL specimens. Sixteen percent (20/125) of paired NP and BAL samples had the same pathogen(s) detected by the FARP, while the remaining $11 \%(14 / 125)$ had discordant results in NP and BAL specimens by the FARP (Table 2). Rhinovirus/enterovirus was the most frequently detected pathogen, being identified in $15.2 \%$ (19/125) of NP swabs and $11.2 \%(14 / 125)$ of BAL specimens. In ICH patients, the overall yield of the FARP was higher in both NP and BAL samples compared with non-ICH patients $(p=0.02)$ (Tables 3 and 4$)$. Twenty-seven $(27 \%)$ of $100 \mathrm{ICH}$ patients had at least one pathogen detected in the NP swab. Of these, 19/27 (70\%) had the same pathogen(s) detected from the paired BAL specimen. The remaining $8(30 \%)$ patients had a negative result in the paired BAL sample. The FARP was positive for at least one pathogen in 24 (24\%) BAL specimens collected from ICH patients, and of these, 19/24 (79\%) had the same pathogen detected in the paired NP swab sample. The remaining $5(21 \%)$ patients had a negative result in the NP specimen (Table 3 ).
In comparison with the FARP, the overall yield of other microbiology studies (e.g., routine bacterial, fungal and viral culture, and individual real-time PCR studies) on the BAL specimens was $29 \%(36 / 125)$ (Table 5). In the ICH group, at least one microbiology study (not including FARP) was positive in $25 \%(25 / 100)$ of the BAL samples, compared to $24 \%(24 / 100)$ of BAL specimens being positive by the FARP. Among $32 \mathrm{ICH}$ patients who had either a positive NP or BAL FARP, 10 (31\%) had additional yield from routine (non FARP) microbiology studies. Among $68 \mathrm{ICH}$ patients who had negative FARP results from both the NP swab and BAL, $16(24 \%)$ had an organism identified by at least one of the routine microbiology tests (Table 5). In non-ICH patients, other microbiology studies were positive for at least one pathogen in $11 / 25$ (44\%) BAL specimens. The majority (92\%; 23/25) of these patients had negative FilmArray results in both NP and BAL samples (Table 5).

In the ICH group, the most common indication for bronchoscopy with BAL in our study was a radiographic infiltrate(s) on chest imaging $(n=83)$, followed by a diagnosis of pneumonia $(n=24)$, cough $(n=22)$, sepsis $(n=21)$, 
TABLE 5: Yield of routine microbiology studies in bronchoalveolar lavage fluid collected from immunocompromised $(n=100)$ and immunocompetent $(n=25)$ patients.

\begin{tabular}{lcc}
\hline Patient group and FilmArray result & Total number & $\begin{array}{c}\text { Number (\%) of BAL fluid samples that were positive } \\
\text { by a routine microbiology test }^{\text {a }}\end{array}$ \\
\hline ICH & 100 & $25^{\mathrm{b}}(25.0 \%)$ \\
Positive FARP & 32 & $10(31.3 \%)$ \\
Negative FARP & 68 & $15(23.5 \%)$ \\
Non-ICH & 25 & $11^{\mathrm{c}}(44.0 \%)$ \\
Positive FARP & 2 & $1(50.0 \%)$ \\
Negative FARP & 23 & $10(43.5 \%)$ \\
\hline
\end{tabular}

No., number; BAL, bronchoalveolar lavage; ICH, immunocompromised host; FARP, FilmArray Respiratory Panel; ${ }^{a}$ routine microbiology testing included Gram staining, fungal smear, acid-fast smear, routine cultures (bacterial, fungal, viral, and mycobacterial), real-time PCR for influenza A/B, respiratory syncytial virus (RSV), Pneumocystis jirovecii, adenovirus, and Legionella spp.; ${ }^{b}$ organisms recovered/detected: Aspergillus spp. $(n=7)$, cytomegalovirus $(n=4)$, Pseudomonas spp. $(n=3)$, Klebsiella spp. $(n=2)$, S. aureus $(n=2)$, methicillin-resistant Staphylococcus aureus $(n=1)$, Serratia marcescens $(n=1)$, Pneumocystis jirovecii $(n=1)$, Scopulariopsis $(n=1)$, Bordetella bronchiseptica $(n=1)$, Haemophilus influenzae $(n=1)$, Stenotrophomonas maltophilia $(n=1)$, Proteus mirabilis $(n=1)$, Mycobacterium avium complex $(n=1)$, and Histoplasma capsulatum $(n=1)$ (three patients had more than one pathogen detected); 'organisms recovered/detected: Mycobacterium avium complex $(n=4)$, Aspergillus spp. $(n=2)$, Staphylococcus aureus $(n=2)$, Stenotrophomonas maltophilia $(n=1)$, Mycobacterium abscessus group $(n=1)$, Achromobacter spp. $(n=1)$, Haemophilus influenzae $(n=1)$, Citrobacter spp. $(n=1)$, and Klebsiella spp. $(n=1)$ (four patients had more than one pathogen detected).

hypoxia $(n=14)$, dyspnea $(n=10)$, upper respiratory tract infection $(n=6)$, or FEV1 (forced expiratory volume in 1 second) decline $(n=2)$. The majority of patients had a clinical and/or radiographic indication for bronchoscopy. Seventytwo $(72 \%) \mathrm{ICH}$ patients and $21(84 \%)$ non-ICH patients had both radiographic and clinical indications for BAL. Ten patients underwent bronchoscopy/BAL for routine surveillance after lung transplant. In one of these patients, human rhinovirus/enterovirus was detected from both the NP and BAL samples. When these surveillance patients (without a clinical or radiographic indication for BAL) were excluded from analysis, the yield of the FARP increased from $27 \%$ $(27 / 100)$ to $28.8 \%(26 / 90)$ in NP swabs and from $24 \%(24 / 100)$ to $25.5 \%(23 / 90)$ in BAL specimens. Out of the $100 \mathrm{ICH}$ patients, 78 had a clinical indication for bronchoscopy/BAL (72 of these also had a radiographic indication) and 22 patients had no clinical indication for bronchoscopy $(12 / 22$ patients had only radiographic indications and 10/22 patients underwent bronchoscopy for routine surveillance after lung transplant). Those patients with a clinical indication for bronchoscopy/BAL were more likely to have a positive FARP result from either the NP or BAL sample compared with those that had no clinical indication for BAL (36\% versus $18 \%$, $p=0.018$ ). Among six patients that had clinical symptoms but no radiographic features of respiratory disease, FilmArray was positive in 3/6 (50\%) patients in either the NP swab or BAL samples. Furthermore, the yield of the FARP was $34.7 \%$ $(25 / 72)$ in patients with both clinical and radiographic indications for bronchoscopy/BAL versus 25\% (3/12) in patients with a radiographic indication alone.

\section{Discussion}

The results of this study suggest that the FARP may be useful in the evaluation of ICH with suspicion of lower respiratory tract infection, as the yield of this test was higher in $\mathrm{ICH}$ (32\%) compared to non-ICH patients $(8 \%) \quad(p=0.02)$ (Tables 2 and 3). Twenty-seven ICH patients (27\%) had a positive NP FARP, with 19/27 (70\%) having the same pathogen(s) detected from the paired BAL sample. The remaining $8(30 \%)$ patients had a negative FARP result in BAL fluid (Table 2). This suggests that if a pathogen is detected from the NP swab by the FARP, performing the multiplex assay on BAL fluid is unlikely to provide additional yield for pathogens included on the FARP. Among the ICH group, 24/100 (24\%) had a pathogen detected from BAL fluid by the FARP. The majority $(79 \% ; 19 / 24)$ of these patients had the same pathogen(s) identified from the NP specimen, while $5(21 \%)$ had a negative FARP result on the NP swab (Table 2). We conclude that if the FARP is negative on a NP swab, there may be additional value of testing BAL fluid by the multiplex assay. It should be noted that regardless of the FARP results on an NP swab, proceeding with bronchoscopy/BAL may still be indicated in $\mathrm{ICH}$ patients with suspected infection since the FARP does not test for certain pathogens (e.g., Aspergillus spp., Pneumocystis, Nocardia spp., and Mycobacteria spp.) that can be important causes of disease in the immunosuppressed population. In support of this, in our cohort, those with a positive FARP on either NP or BAL samples had an additional pathogen(s) detected by routine microbiology studies in $31 \%$ of cases. Furthermore, among those patients with negative FARP results on both NP and BAL specimens, there was an additional yield from routine microbiology studies on the BAL fluid in $23.5 \%$ of cases (Table 5).

The yield of routine microbiology testing (e.g., cultures and individual real-time PCR assays) in the ICH cohort was $25 \%$, compared to the FARP being positive in $27 \%$ of NP swabs and $24 \%$ of BAL specimens. These results suggest that the FARP assay may serve as a rapid alternative to routine testing for those targets that are included on the multiplex panel. Furthermore, FARP testing may play an important role in patient management decisions, including infection control and antimicrobial stewardship [11]. If a bacterial pathogen is detected, broad-spectrum coverage could be modified to offer more specific antimicrobial therapy. In contrast, if a viral pathogen is detected and bacterial cultures remain negative, it may be prudent to discontinue antibiotics and initiate targeted antiviral therapy [11]. Bronchoscopy with BAL is routinely ordered in $\mathrm{ICH}$ patients at our 
institution. Our study showed that standard microbiology studies on BAL fluid had a yield of $25 \%$ in this cohort, which is comparable to the yield of the FARP (24\%). We believe that the comparable yield of FARP in this population provides additional support for this noninvasive test as a reasonable adjunct to BAL testing in the $\mathrm{ICH}$.

Many ICH patients with respiratory illness are placed on empiric, broad-spectrum antimicrobial therapy before the BAL is collected, which can confound the yield of BAL cultures. Multiplex molecular testing, however, may not be affected by the prior administration of antibiotics. We propose that when used in conjunction with routine microbiology studies, FARP testing on BAL fluid may have a significant impact on the diagnosis and management of ICH patients with respiratory illnesses.

Conversely, FARP appears to be less useful in non-ICH patients. In our study, the yield of routine microbiology studies in non-ICH patients was higher than that in ICH patients (44\% versus $25 \%$, resp.). However, the FARP showed a lower yield when compared to routine cultures in the non-ICH cohort ( $8 \%$ versus $32 \%$, resp.).

The performance of the FARP observed in our study is comparable with other studies in the literature. Our group previously performed a retrospective review of FARP testing on NP and BAL samples collected within one week of each other. This study reported the FARP to be positive in $20.9 \%$ $(18 / 86)$ of NP swabs versus $37.2 \%$ (32/86) of BAL specimens [10]. The higher yield from BAL is likely due to the fact that only patients with a radiographic and/or clinical indication for BAL were included.

Hammond et al. [2] also assessed the performance of the FARP using NP swabs $(n=34)$ and BAL specimens $(n=56)$ collected from ICH patients $(n=87)$. Importantly, this study did not compare NP swabs and BAL fluid that were collected concurrently. The results suggested that the FARP had an overall yield of $29 \%$ and identified more respiratory pathogens compared to conventional methods (direct fluorescent antibody (DFA), enzyme immunoassays, and viral culture) [2].

Soccal et al. [7] assessed the detection of respiratory viruses by individual real-time PCR assays using NP and BAL samples collected concurrently from lung transplant patients. They observed an overall viral positivity rate of $29.3 \%$ in NP samples and $17.2 \%$ in BAL samples. They also demonstrated that lung transplant patients had a much slower lung function recovery if they were found to have evidence of rejection in addition to viral pathogen detection, providing important prognostic information [7]. The lower yield of PCR in BAL specimens could be explained by the inclusion of any lung transplant patient that underwent bronchoscopy, without necessarily having respiratory symptoms, pulmonary infiltrates, or decline in airflow. Our data supports the notion that those without clinical or radiographic indications for BAL have a lower rate of pathogen detection.

This study has several limitations that should be discussed. First, the number of NP $(n=28)$ and BAL $(n=26)$ samples testing positive by the FARP was relatively low, so this limits the conclusions that can be made regarding specific pathogens tested. Second, due to the heterogeneous radiographic and clinical findings in these patients, it was difficult to identify specific clinical or radiologic features that would prompt the performance of FARP testing of BAL fluid versus a NP swab alone. Despite these limitations, this is the first study to compare the performance of the FARP using BAL fluid and NP swabs collected concurrently from ICH patients and a non-ICH comparison group.

When the FARP is performed in conjunction with routine microbiology tests in the $\mathrm{ICH}$, it can provide important diagnostic information, especially in patients with clinical manifestations of respiratory illness. Our data suggest that, in this cohort, FARP testing should be initially performed on a NP swab. If positive, there is limited additional yield of testing the FARP on BAL fluid. However, if the results of the multiplex assay are negative on the NP swab, testing a BAL specimen may provide additional information, especially in patients with lower respiratory tract disease. It is necessary to highlight that the FARP does not cover all causes of respiratory infection, and therefore, routine microbiology testing (e.g., bacterial, mycobacterial, and fungal cultures) will continue to be essential.

In conclusion, respiratory infections that are generally well tolerated in the immunocompetent host can be serious and life-threatening in the ICH. The early detection, diagnosis, and appropriate management of these infections can have a substantial impact on clinical care and outcomes. Therefore, multiplex testing may play an important role in the management of $\mathrm{ICH}$ presenting with respiratory symptoms.

\section{Disclosure}

All FilmArray Respiratory Panel reagents were purchased using Collaborative Research Grant Funds supplied by the Department of Laboratory Medicine and Pathology at Mayo Clinic.

\section{Conflicts of Interest}

The authors claim no conflicts of interest related to the products used in this study.

\section{Acknowledgments}

The authors would like to thank the following people for their technical assistance in this study: Emily Fernholz, Aimee Boerger, and Corrisa Miliander from the Department of Laboratory Medicine and Pathology and Kathleen Mieras, Pulmonary Clinical Research Coordinator, Mayo Clinic Rochester, MN, USA.

\section{References}

[1] M. R. Couturier, T. Barney, G. Alger et al., "Evaluation of the FilmArray ${ }^{\varpi}$ respiratory panel for clinical use in a large children's hospital," Journal of Clinical Laboratory Analysis, vol. 27, no. 2, pp. 148-154, 2013.

[2] S. P. Hammond, L. S. Gagne, S. R. Stock et al., "Respiratory virus detection in immunocompromised patients with FilmArray 
respiratory panel compared to conventional methods," Journal of Clinical Microbiology, vol. 50, no. 10, pp. 3216-3221, 2012.

[3] C. P. Layman, S. M. Gordon, D. U. Elegino-Steffens, W. Agee, J. Barnhill, and G. Hsue, "Rapid multiplex PCR assay to identify respiratory viral pathogens: moving forward diagnosing the common cold," Hawaii Journal of Medicine \& Public Health, vol. 72, no. 9, pp. 24-26, 2013.

[4] M. J. Loeffelholz, D. L. Pong, R. B. Pyles et al., "Comparison of the FilmArray respiratory panel and Prodesse real-time PCR assays for detection of respiratory pathogens," Journal of Clinical Microbiology, vol. 49, no. 12, pp. 4083-4088, 2011.

[5] V. M. Pierce, M. Elkan, M. Leet, K. L. McGowan, and R. L. Hodinka, "Comparison of the Idaho Technology FilmArray system to real-time PCR for detection of respiratory pathogens in children," Journal of Clinical Microbiology, vol. 50, no. 2, pp. 364-371, 2012.

[6] K. H. Rand, H. Rampersaud, and H. J. Houck, "Comparison of two multiplex methods for detection of respiratory viruses: FilmArray RP and xTAG RVP," Journal of Clinical Microbiology, vol. 49, no. 7, pp. 2449-2453, 2011.

[7] P. M. Soccal, J. D. Aubert, P. O. Bridevaux et al., "Upper and lower respiratory tract viral infections and acute graft rejection in lung transplant recipients," Clinical Infectious Diseases, vol. 51, no. 2, pp. 163-170, 2010.

[8] P. Ruggiero, T. McMillen, Y. W. Tang, and N. E. Babady, "Evaluation of the BioFire FilmArray respiratory panel and the GenMark eSensor respiratory viral panel on lower respiratory tract specimens," Journal of Clinical Microbiology, vol. 52, no. 1, pp. 288-290, 2014.

[9] F. V. Fossella, R. DeVore, R. N. Kerr et al., "Randomized phase III trial of docetaxel versus vinorelbine or ifosfamide in patients with advanced non-small-cell lung cancer previously treated with platinum-containing chemotherapy regimens. The TAX 320 Non-Small Cell Lung Cancer Study Group," Journal of Clinical Oncology, vol. 18, no. 12, pp. 2354-2362, 2000.

[10] N. Azadeh, K. K. Sakata, A. M. Brighton, H. R. Vikram, and T. E. Grys, "FilmArray respiratory panel assay: comparison of nasopharyngeal swabs and bronchoalveolar lavage samples," Journal of Clinical Microbiology, vol. 53, no. 12, pp. 37843787, 2015.

[11] S. C. Chiu, Y. C. Lin, H. C. Wang et al., "Surveillance of upper respiratory infections using a new multiplex PCR assay compared to conventional methods during the influenza season in Taiwan," International Journal of Infectious Diseases, vol. 61, pp. 97-102, 2017. 


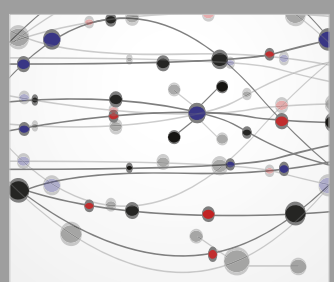

The Scientific World Journal
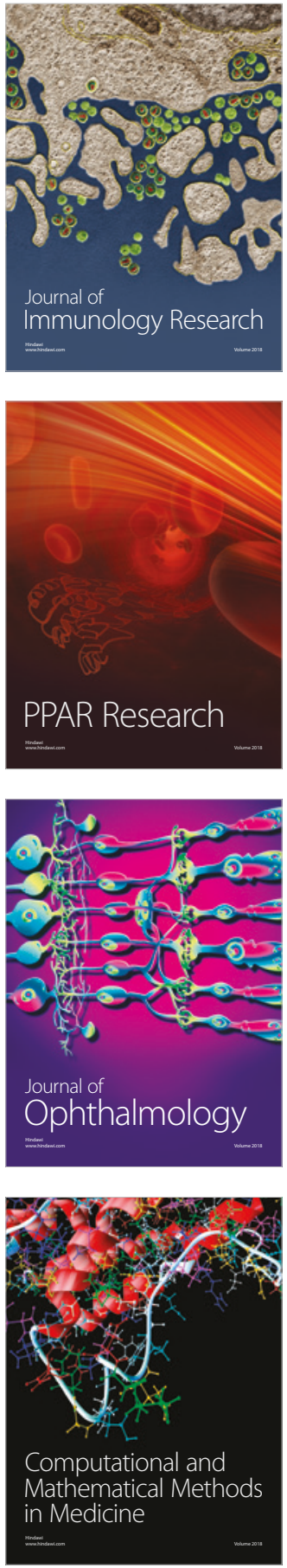

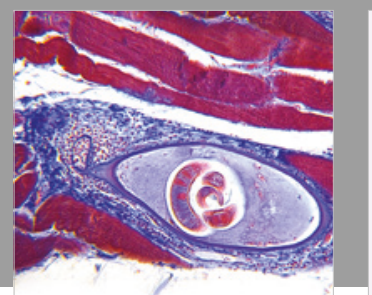

Gastroenterology Research and Practice

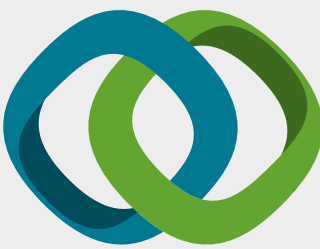

\section{Hindawi}

Submit your manuscripts at

www.hindawi.com
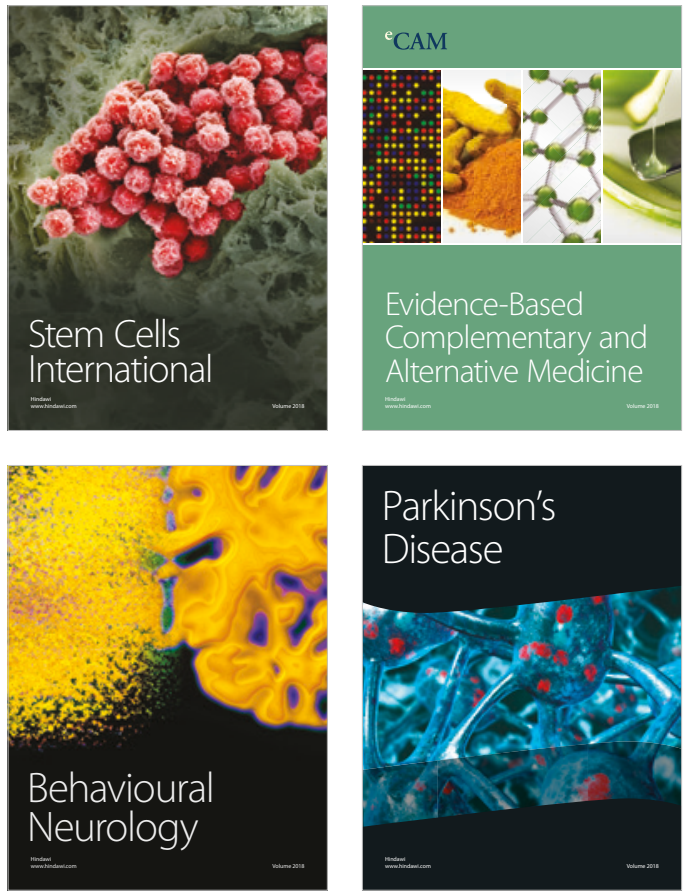

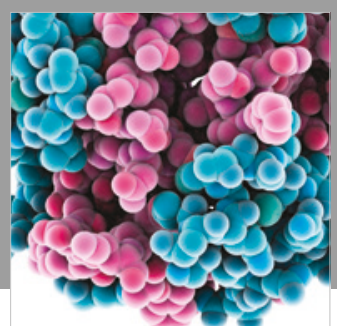

ournal of

Diabetes Research

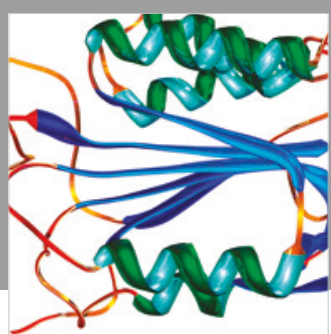

Disease Markers
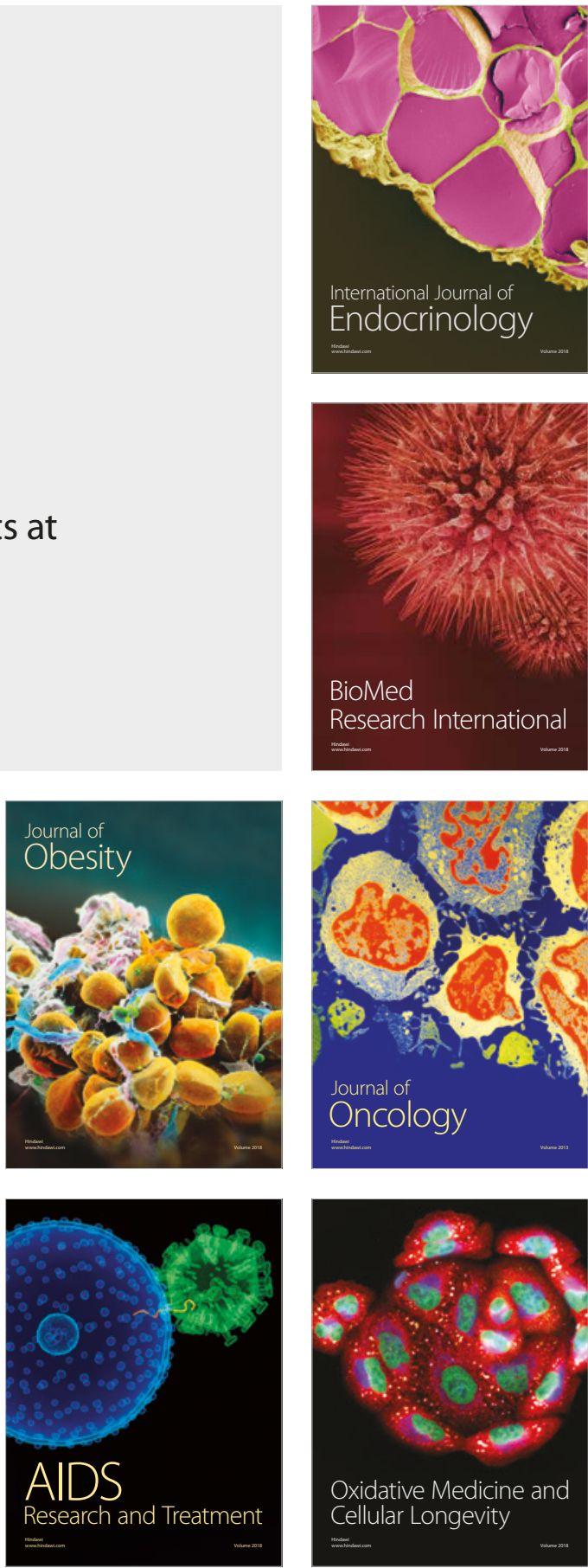